\title{
Round table discussion of session F: convection and rotation
}

\author{
Ian W. Roxburgh ${ }^{1,2}$ \\ ${ }^{1}$ Astronomy Unit, Queen Mary, University of London, Mile End Road, London E14NS, UK. \\ ${ }^{2}$ Observatoire de Paris, Place Jules Janssen, 92195 Meudon, France. \\ email: i.w.roxburgh@qmul.ac.uk
}

\begin{abstract}
The Session F round table. and audience, considered the following questions:
(i) what determines the differential rotation in stellar convective zones?

(ii) If there really is a rapid inward increase in angular velocity in giants will it have significant effects in the late stages of stellar evolution?
\end{abstract}

(iii) How effective is penetration above a convective core?

(iv) Why is the solar core nearly uniformly rotating?

\section{What determines the differential rotation in stellar convective zones?}

Ian Roxburgh. We have seen that as determined from helioseismology the angular velocity in the solar convective zone rotation is almost constant on radial lines, and from simulations of red giants that the angular velocity increases inwards. Have people any thoughts as to what causes the differential rotation to behave in the way it does.

Matthias Rempel considered that the deviation from the Taylor Proudman state is caused by latitudinal variations in the entropy between pole and equator one possibility being the anisotropic heat transport caused by the interaction of rotation and convection, an other being the tachocline at the base of the convective zone leads to latitudinal variations in entropy since this has the largest deviation from the Taylor Proudman state. One needs a temperature variation between pole and equator of the order of $6^{\circ} \mathrm{K}$ between pole an equator to obtain a differential rotation profile which is close to the observed one.

Ian Roxburgh. What about deep convective envelopes in giants? Do you have any explanation of your numerical results that the angular velocity increases inwards so rapidly? I remember models by Biermann and Kippenhahn from many years ago that were based an models of anisotropic turbulence.

\begin{abstract}
Ana Palacios responded that she didn't have an explanation but stressed that her results were consistent with observations of the angular velocities of stars on the main sequence and horizontal branch which suggest that at some stage stars build up a reservoir of angular momentum in the interior of the star, since stars on the horizontal branch are rotating faster than would otherwise be the case. An interesting question is at what point in the evolution of a star does the angular velocity profile change from being solar like to that found in her computations for giants - this is likely to be important in understanding abundance anomalies.
\end{abstract}


Juri Toomre agreed that the we should not say that this is so because our computations give these results and stressed that from computations we have learned that coherent structures emerge with alignments etc., but it is difficult to say what causes them. But these are big players for angular momentum and anisotropic heat transport - the Reynold's stresses are a big player in this game. As resolution in numerical simulations has improved we find long lived coherent structures spanning over many scale heights and they are the dominant features.

Nick Brummel agreed with Juri and Mathias, that recent simulations have shown that latitudinal entropy variations can give the solar differential rotation, though it is not self consistently imposed, As Juri says the Reynold's stresses are dominated by coherent structures but it is very hard to extract a simple theory from the very complicated output of these numerical simulations.

Vittorio Canuto suggested that one could approach the problem in the opposite direction. That is since we have the beautiful results on solar differential rotation reported by Christensen-Dalsgaard, one could take this as given and seek to determine the Reynold's stresses, and compare this with the simulations.

Sasha Brun stated that they found that when they imposed a latitudinal temperature difference of $8-10^{\circ} \mathrm{K}$ in their recent 3 -D simulations they were able to get an almost conical angular velocity variation as deduced from helioseismology. But the urged caution as we don't really know what causes this pole equator entropy variation. What they found was that i some models they found the rotation was almost cylindrical. He reported that he had used 3-D simulations to compute all the terms in the thermal wind balance, not just the baroclinic term. that is Reynold's stresses, Maxwell stresses, etc., and that he is trying to do is to explore parameter space to find what combination of parameters (Peclet number, Rayleigh number, etc) will give $8-10^{\circ} \mathrm{K}$ and conical variation in the angular velocity. He stressed that tilting plumes heat transport clearly play an important role.

Ian Roxburgh provocatively observed that he inferred from what Sasha had said that he could not answer the question he originally posed to the meeting - but that he was working on it.

Sasha Brun responded that this was not quite fair - we can say the about a $8^{\circ} \mathrm{K}$ latitudinal variation gives a conical variation in angular velocity but we cannot say why it is 8 and not 6 or 15 .

\section{Rapidly rotating cores and late stages of stellar evolution}

Ian Roxburgh then posed the second question to the meeting:

If there really is a rapid inward increase in angular velocity in giants will it have significant effects in the late stages of stellar evolution? The results presented by Palacios gave an inward increase $\propto 1 / r^{2}$ or $\propto 1 /(r \sin \theta)^{2}$. This would suggest that stellar cores could be very rapidly rotating in the later stages of evolution, is this likely to important?

Dave Arnett responded that he thought it definitely would, even if it doesn't do something dramatic like make the core into a disc. it would have an important effect on mixing. A key part in the physics of core collapse is the size of the core so the combination of core size and rotation gives a wide parameter space for the collapse modellers to explore. He 
also pointed out that it would have important consequences in more mundane stages of stellar evolution which we can observe in more detail, such as abundances at the surface of stars.

Ian Roxburgh informed the meeting that in the 1940s Fred Hoyle had proposed a model of Cepheid variables in which a rapidly rotating core had split into two stars, and that a Cepheid was this compact binary system surrounded by a common envelope.

A $\mathbf{N}$ Other member of the audience stressed the importance of aligned structures in determining the Reynold's stresses. In the early DNS simulations of isotropic turbulence these structures or "worms" were observed with widths down to the Kolmogorov scale, or the resistive scale in the case of MHD turbulence. In his view it is critical that we are able to model all the scales down to the Kolmogorov scale in our numerical simulations in order to compute the Reynold's stresses correctly.

\section{Overshooting from stellar cores}

Ian Roxburgh then raised the next topic: convective overshooting or penetration above a convective core, stating the importance of such overshooting not only for the main sequence phase but also for its imprint on subsequent phases of stellar evolution. He remarked that there was some disagreement on the effectiveness of such overshooting/penetration and invited the panel and audience to address this issue, in particular was overshooting more effective than undershooting?

Nick Brummel responded that some people have done some work on this but he didn't remember that overshooting was any more effective than undershooting.

Sasha Brun reported that they had undertaken simulations of overshooting in a model of a main sequence F star. They distinguish between overshooting which modifies the adiabatic structure, and penetration beyond the overshoot layer which is effective in mixing chemical composition. They found that the overshoot region had a strong latitudinal variation but that the surrounding penetrative region was round. But they did not have a chemical composition gradient in their computations.

\section{Why is the solar core nearly uniformly rotating?}

Ian Roxburgh then observed that we were running out of time but had one other question which may be should perhaps be left to the next session - that is why is the angular velocity in the solar interior almost uniform - is it just simply due to a magnetic field? This elicited three comments:

(i) Yes. (ii) No. (iii) Maybe.

With that the session was called to a close. 